\title{
A Study of Coconut (Cocos Nucifera L.) Phosphatidylcholine Species
}

\author{
DWI HUDIYANTI ${ }^{1 *}$, MARLIANA JAYANTI ${ }^{2}$, MUHAMMAD FUAD AL KHAFIZ ${ }^{3}$, \\ and KHAIRUL ANAM ${ }^{1}$
}

\begin{abstract}
'Department of Chemistry, Faculty of Science and Mathematics, Diponegoro University - Jl. Prof. Soedarto, SH 50275 Semarang, Indonesia.

${ }^{2}$ Chemistry Program, Faculty of Science and Mathematics, Diponegoro University - Jl. Prof. Soedarto, SH 50275 Semarang, Indonesia.

${ }^{3}$ Post Graduate Program, Faculty of Science and Mathematics, Diponegoro University - Jl. Prof. Soedarto,

SH 50275 Semarang, Indonesia.

*Corresponding author E-mail: dwi.hudiyanti@live.undip.ac.id
\end{abstract}

http://dx.doi.org/10.13005/ojc/340636

Received: October 31, 2018; Accepted: November 29, 2018)

\begin{abstract}
Coconut (Cocos nucifera L.) phosphatidylcholines, or CocoPCs species are studied in this paper. CocoPCs is fractionated from coconut phospholipids (CocoPLs) using methanol eluent in silica column chromatography. Analysis of the CocoPCs, by FTIR, GCMS and LCMS, reveals that among the isolated CocoPCs species are 16:0/18:0-PC with $\mathrm{m} / \mathrm{z} 763$ at $\mathrm{Rf} 6.02$ and 18:1-LysoPC with $\mathrm{m} / \mathrm{z} 522$ at $\mathrm{R}_{f} 4.93$. The composition of CocoPCs fatty acid chain residues are Dodecanoic acid (C12:0), Tetradecanoic acid (C14:0), 9-Hexadecenoic acid (C16:1), Hexadecanoic acid (C16:0), 9, 12-Octadecadienoic acid (Z,Z) (C18:2), 9-Octadecenoic acid (C18:1) and Octadecanoic acid (C18:0). The CocoPCs content is $6.317 \%$ of $100 \mathrm{mg}$ CocoPLs.
\end{abstract}

Keywords: Drug delivery systems, Phospholipid, Amphiphilic, Fatty acid, Natural membrane.

\section{INTRODUCTION}

Lipid based drug delivery systems are one of many alternatives to overcome various problems related to drug formulation and application. These systems have been known to provide many benefits, including increased bioavailability and versatility of drugs. Their abilities are proven in a variety of treatment methods and they are safe and efficient. The lipids that are widely used in these systems are phospholipids'.
Phospholipids, which are structural and functional lipids, generally contain two distinct molecular parts. One part is polar and the other contains nonpolar parts that are bound to the glycerol framework. The combination of polar and nonpolar segments in these molecules is called amphiphilic; which illustrates the tendency of molecules to be in hydrophilic and lipophilic phase interfaces. The hydrophilic portion consists of phosphate esters, while the lipophilic portion consists of two fatty acids chains.

This is an Open Access article licensed under a Creative Commons license: Attribution 4.0 International (CC- BY). Published by Oriental Scientific Publishing Company @ 2018

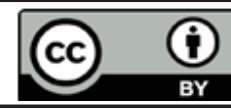


This amphiphilic characteristic plays an important role in its use as a drug delivery system².

Various types of hydrophilic and lipophilic groups, found in phospholipids, provide various phospholipid species with various amphiphilic properties. The molecular species, phospholipids, are phosphatidylcholine (PC) lipids, phosphatidylserine (PS), phosphatidylethanolamine (PE), phosphatidylglycerol (PG), and phosphatidilinositol (PI). PC and PE are neutral molecules (zwitter ion), while PS, PG and PI are charged phospholipids. Amine-bound phosphate groups; often called nitrogen bases, are serine, choline or ethanolamine. Types of polar groups, the length of the fatty acid chain and their degree of saturation (i.e., number of double chains) determines the physicochemical and functional properties of phospholipids ${ }^{3,4}$. This affects the capacity and ability of phospholipids to bind active ingredients and drugs when used as drug delivery systems.

The main sources of phospholipids in nature are plants and animals. Phospholipids are the main constituent of cell membranes; namely lipid bilayers ${ }^{5,6}$. Phospholipids in natural membranes usually contain two different chains; and usually, one of them is unsaturated. Natural phospholipids that have been used as current drug delivery systems, phosphatidylcholine (PC), are generally derived from eggs and soybean with their fatty acid chains ranging from C16:0-C22:6 to C14:0-C20:07-9. With the growing need for phospholipids as drug delivery systems, we should seek other sources of phospholipids to fulfil this demand.

Research by Hudiyanti et al., ${ }^{10}$ used methanol eluent in coconut crude lipids to obtain Coconut phospholipids (CocoPLs). Coconut phospholipids contain phosphatidylcholine (CocoPCs), phosphatidylserine (cocoPSs), and/or phosphatidylethanolamine (cocoPEs) species with the lipophilic groups of dodecanoic acid (C12:0), octadecanoic acid (C18:0) and hexadecanoic acid (C16:0), which are abundant in CocoPLs ${ }^{3}$. In this paper, we continue the study by analyzing the CocoPCs species and the lipophilic constituents of CocoPCs. Our new finding is that CocoPCs content is $6.317 \%$ in $100 \mathrm{mg}$ CocoPLs. The species contained in isolated CocoPCs include 16:0/18:0-PC with $\mathrm{m} / \mathrm{z} 763$ at $R_{f} 6.02$ and 18:1-LysoPC with $\mathrm{m} / \mathrm{z}$ 522 at $R_{f} 4.93$. The fatty acid chain residues are (C 16:0) palmitic acid, (C 18:0) stearic acid and (C 18:1) elaidic acid. This finding exposes that the CocoPCs has fatty acid chain residues similar to soybean PCs so that it is expected to have potency as raw material for a drug delivery system.

\section{EXPERIMENITAL}

\section{Materials}

Coconut meat (Cocos nucifera L.) was purchased from a local market. Chloroform, methanol, ethanol, n-hexane, TLC-silica gel plate 60 GF254, silica gel $60(0.2-0.5 \mathrm{~mm})$, acetone, acetic acid, diethyl ether and Dragendorff reagent were purchased from Merck.

\section{Isolation of (CocoPLs)}

Isolation of CocoPLs from dried coconut powder was performed according to the method presented by Hudiyanti et al., ${ }^{10,11}$. Briefly, the technique was as follows: dried coconut powder was macerated with a chloroform and methanol mixture $(2: 1 \mathrm{v} / \mathrm{v})$. The filtrate was purified further by partitioning in $n$-hexane and $80 \%$ ethanol layers, followed by evaporation of the polar layer to obtain CocoPLs.

\section{Fractionation of CocoPCs}

To acquire coconut phosphatidylcholines (CocoPCs), we fractionated CocoPLs as follows: A total of $100 \mathrm{mg}$ of CocoPLs was dissolved in 2 $\mathrm{ml}$ of chloroform. The CocoPLs solution was then fractionated by chromatography over silica gel 60 (0.2-0.5 mm) using several mobile phases; namely chloroform, chloroform/methanol $(6: 1, \mathrm{v} / \mathrm{v})$, acetone/ methanol $(9: 1, \mathrm{v} / \mathrm{v})$, and methanol.

A total of $6 \mathrm{~g}$ of silica gel $60(0.2-0.5 \mathrm{~mm})$ was dissolved in $15 \mathrm{~mL}$ of chloroform to form a silica slurry. The silica slurry was then inserted into a chromatography column until it was solid and compact without holes and cracks. A total of $100 \mathrm{mg}$ of CocoPLs was dissolved in $2 \mathrm{~mL}$ of chloroform. The CocoPLs solution was then poured into the column. Next, $100 \mathrm{~mL}$ of mobile phase was eluted; starting with a low polarity phase and then continuing with a high polarity phase i.e., chloroform, chloroform/ methanol $(6: 1, \mathrm{v} / \mathrm{v})$, acetone/methanol $(9: 1, \mathrm{v} / \mathrm{v})$, and 
methanol. The fractions were collected and identified by thin layer chromatography (TLC) under a UV lamp of $265 \mathrm{~nm}$ and $365 \mathrm{~nm}$. The spotting reagent used for the choline species was Dragendorff's reagent.

The fraction with choline species was evaporated at $40^{\circ} \mathrm{C}$ until a gel solid was formed. The gel solid was characterized using FTIR spectrometry to identify its functional group. In order to resolve the inherently coincided bands in the FTIR spectra we used Fityk program for deconvolution technique ${ }^{12,13}$. GC-MS chromatography was used to determine acyl chain fatty acid compounds. GC-MS analysis of the CocoPCs was performed using a GCMS-QP2010S SHIMADZU system equipped with an AGILENTJ\%W HP-5 column ( $30 \mathrm{~m} \times 0.25 \mathrm{~mm}$ ). An electron ionization system was operated in electron impact mode with an ionization energy of $70 \mathrm{eV}$. Helium gas $(99.999 \%)$ was used as a carrier gas at a total flow rate of $80.1 \mathrm{~mL} / \mathrm{min}$, column flow of $0.54 \mathrm{~mL} / \mathrm{min}$, linear velocity $26.7 \mathrm{~cm} / \mathrm{sec}$, purge flow $3.0 \mathrm{~mL} / \mathrm{min}$ and split ratio 141.6. The column oven temperature was $50.0^{\circ} \mathrm{C}$ and injection temperature was $300.00^{\circ} \mathrm{C}$. The gas flow was controlled with pressure of $13.0 \mathrm{kPa}$. LC-MS chromatography with TOF MS ES+ BPI method was performed to determine the molecular weight and structure of the phospholipid compounds of the choline species.

\section{RESULTS AND DISCUSSION}

\section{Isolation of CocoPCs}

Dragendorff's reagent detects tertiary amines; and was therefore utilized to detect choline species of CocoPLs viz. CocoPCs. TLC analysis of CocoPLs fractionations showed that CocoPCs was found in methanol fraction. The chloroform, chloroform/methanol $(6: 1, \mathrm{v} / \mathrm{v})$, and acetone/ methanol $(9: 1, v / v)$ fractions did not reveal any spots; either under UV lamp or after being sprayed with Dragendorff's reagent. The methanol fraction showed development of an orange coloured spot at $R_{f} 0.63$ under Dragendorff's reagent; thus indicating the presence of choline species, CocoPCs (see Fig. 1). After evaporation of the methanol fraction, the CocoPCs content was $6.317 \%$ per $100 \mathrm{mg}$ CocoPLs (Fig. 2).

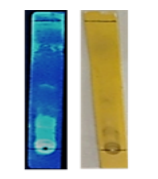

a. Chloroform fraction

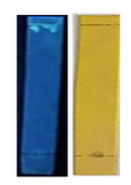

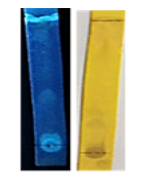

b. Chloroform/methanol (6:1) fraction

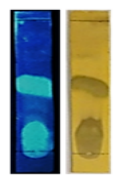

d. Methanol fraction

Note: Blue colour was under uv lamp $\lambda=365 \mathrm{~nm}$, brown colour was after sprayed by dragendorff's reagent

Fig. 1. TLC spot of CocoPLs fractions

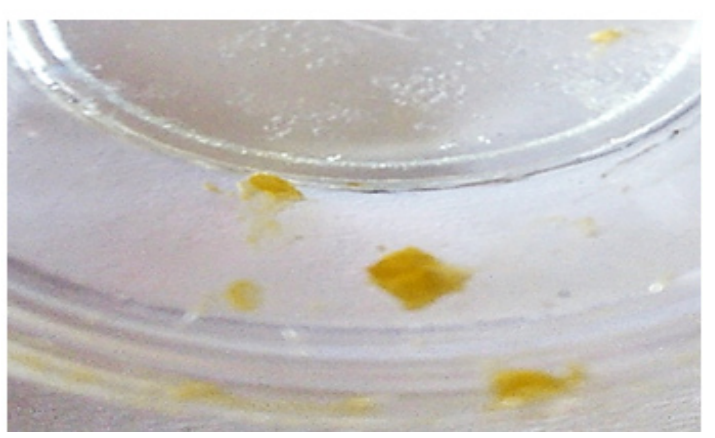

Fig. 2. CocoPCs

The CocoPCs was characterized by FTIR (Fig. 3). The FTIR spectra of CocoPCs (Fig. 3) display a lot of overlapping peaks especially around the phospholipid related absorption regions ${ }^{14}$ hence we need to improve the resolution of the spectra at this regions. The enhanced resolution was obtained by peak deconvolution ${ }^{12}$ on the important regions; specifically, the phosphate and tertiary amine functional group regions (Fig. 4).

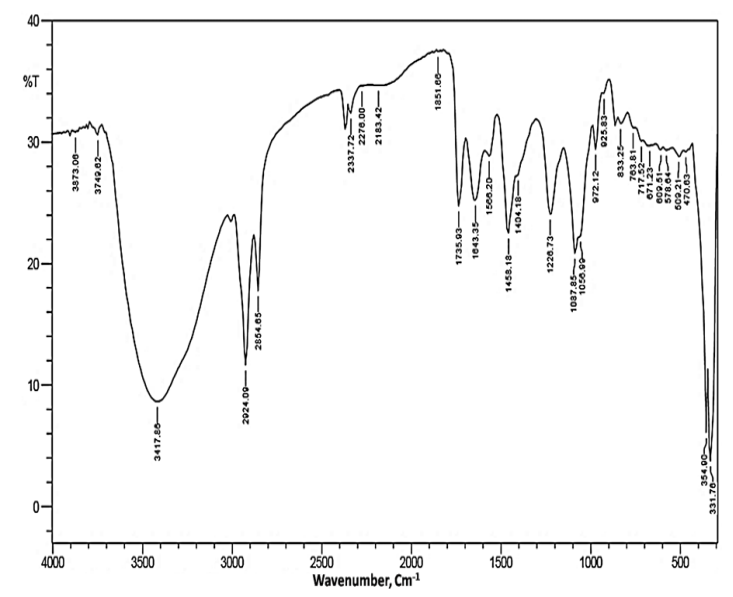

Fig. 3. FTIR spectra of CocoPCs 

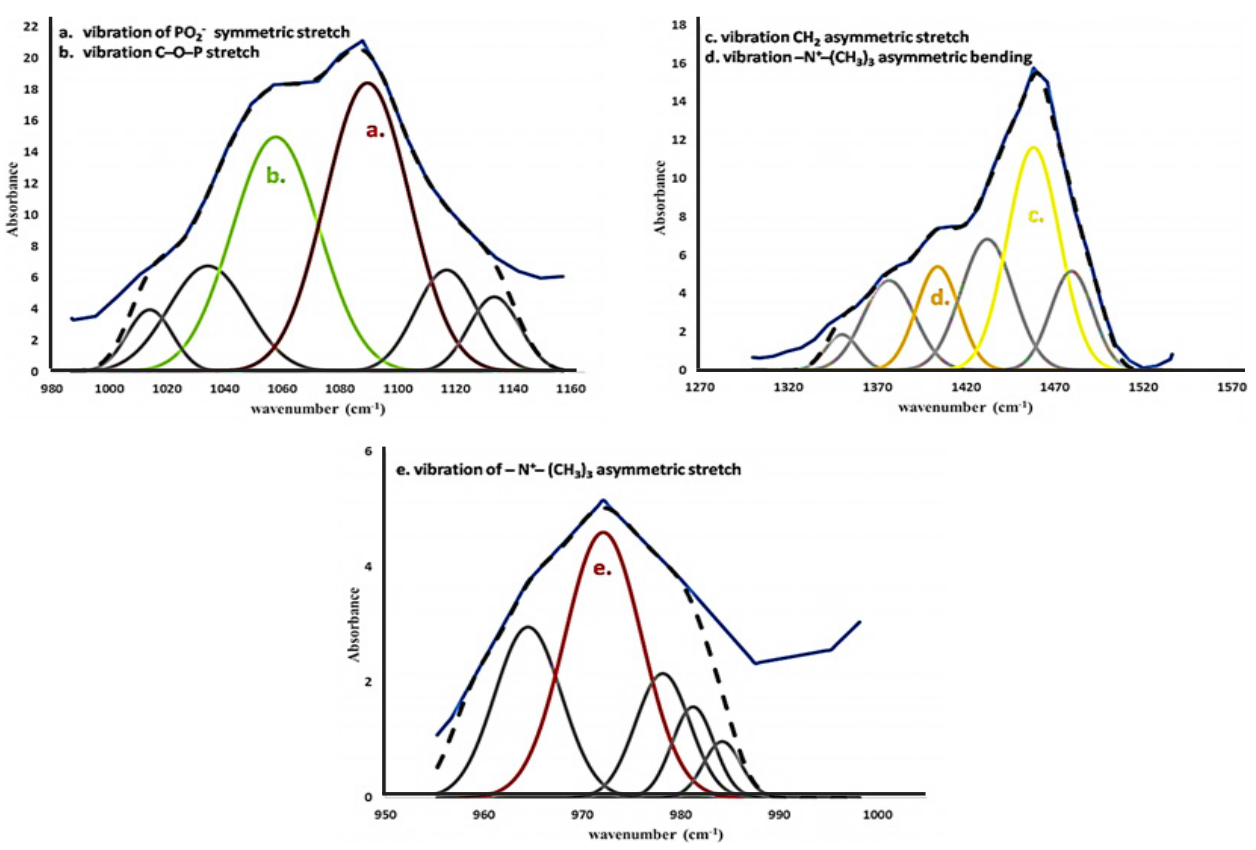

Fig. 4. FTIR Peak deconvolution on the CocoPCs functional groups

Fig. 4 shows deconvolution peaks for phosphate and choline groups of CocoPCs. Peaks $a$ and $b$ show the vibration of the phosphate group, while $\mathrm{c}, \mathrm{d}$ and e are for the choline group. Peak a indicates the symmetry stretch vibration of $\mathrm{PO}_{2}^{-}$at $1087.85 \mathrm{~cm}^{-1}$ and peak $\mathrm{b}$ indicates the presence of vibration C-O-P stretch at $1056.99 \mathrm{~cm}^{-1} .{ }^{12}$ Peaks c, d, and e point towards a vibration $\mathrm{CH}_{2}$ stretch asymmetry at $1458.18 \mathrm{~cm}^{-1}, \mathrm{~N}^{+}-\left(\mathrm{CH}_{3}\right)_{3}$ bending asymmetry at $1404.18 \mathrm{~cm}^{-1}$ and $\mathrm{N}^{+}-\left(\mathrm{CH}_{3}\right)_{3}$ stretch asymmetry at $972.12 \mathrm{~cm}^{-1}$, respectively.

The FTIR spectra of methanol fraction, along with peak deconvolution analysis of CocoPCs, confirmed that the fraction contained phosphatidylcholine i.e., CocoPCs. This was indicated by specific peak absorption of phosphatidylcholine functional groups viz. alkyl, carbonyl, phosphate and quaternary amines $\left(\mathrm{N}^{+}-\left(\mathrm{CH}_{3}\right)_{3}\right)$ at two specific areas, $1404.18 \mathrm{~cm}^{-1}$ and $972.12 \mathrm{~cm}^{-1}$, respectively. Functional groups of CocoPCs are presented in Table 1.

Fatty acid residues composition of CocoPCs was analysed by GC-MS. The chromatogram of fatty acid methyl ester of CocoPCs is presented in Fig. 5. The fatty acid chain residues found in CocoPCs, which have a peak area of more than $10 \%$, is presented in Table 1 . The results confirm similarity between the fatty acid residues found in CocoPCs with the one in coconut lipids ${ }^{10}$.
Characterization of CocoPCs, using LC-MS with positive ion mode, resulted in the chromatogram shown in Fig . 6. The CocoPCs peak found at $\mathrm{R}_{f} 6.02 \mathrm{~min}$, with the product ion spectrum of deprotonated molecular ion, are $\mathrm{m} / \mathrm{z}$ 763. The mass spectrum revealed that the dominant fatty acid chain residues in CocoPCs are palmitic acid (C 16:0) and stearic acid (C 18:0). These fatty acid chains are in agreement with the GC-MS mass spectrum shown in Table 2. The chromatogram also revealed that the CocoPCs still had a low purity; since more peaks existed. Furthermore, the peak at the retention time of $4.93 \mathrm{~min}$ had the highest percentage. This peak, with $\mathrm{m} / \mathrm{z}$ 522, was lysophosphatidylcholine with elaidic fatty acid chain i.e., 18:1-LysoPC.

Table 1: Functional groups of CocoPCs

\begin{tabular}{cc}
\hline Wavenumber, $\mathrm{cm}^{-1}$ & Functional groups \\
\hline 3417.68 & $\mathrm{~N}-\mathrm{H}$ asymmetric stretch \\
2924.09 & $\mathrm{C}-\mathrm{H} \mathrm{sp}{ }^{3}$ asymmetric stretch \\
2854.65 & $\mathrm{C}-\mathrm{H} \mathrm{sp}^{3}$ symmetric stretch \\
1735.93 & $\mathrm{C}=\mathrm{O}$ stretch (esters) \\
1566.20 & $\mathrm{CO}_{2}^{-}$asymmetric stretch \\
1458.18 & $\mathrm{CH}_{2}$ asymmetric stretch \\
1404.18 & $\mathrm{~N}^{+}-\left(\mathrm{CH}_{3}\right)_{3}$ asymmetric bending \\
1087.85 & $\mathrm{PO}_{2}^{-}$symmetric stretch \\
1056.99 & $\mathrm{C}-\mathrm{O}-\mathrm{P}$ stretch \\
972.12 & $\mathrm{~N}^{+}-\left(\mathrm{CH}_{3}\right)_{3}$ asymmetric stretch \\
\hline
\end{tabular}




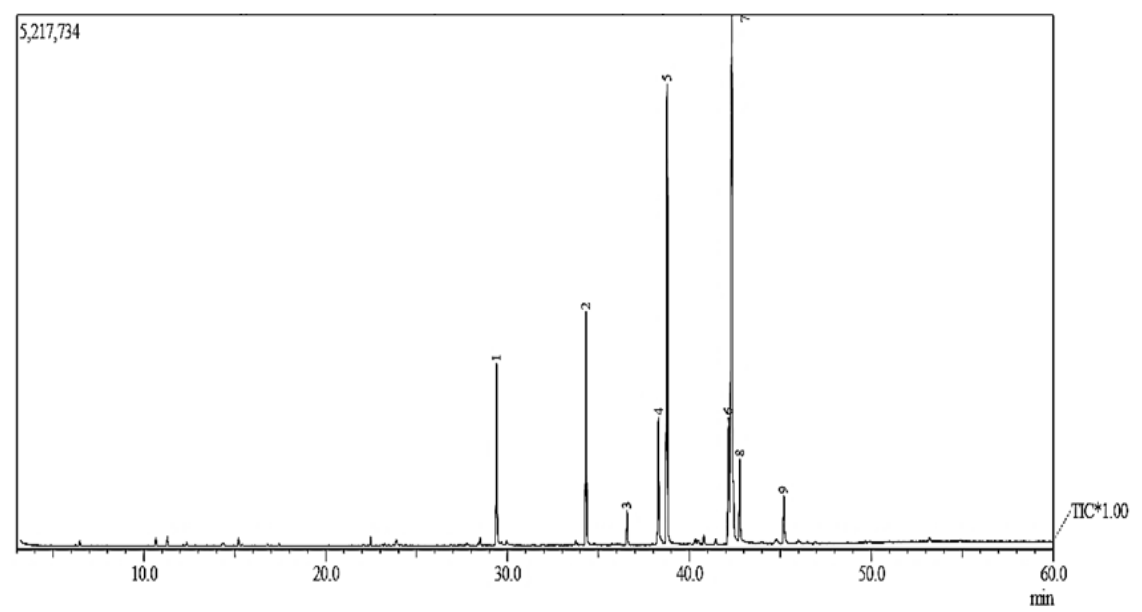

Fig. 5. Chromatogram of CocoPCs

Table 2: Fatty acid chain residues of CocoPCs from GC-MS analysis

\begin{tabular}{cccc}
\hline Peak & $\mathrm{t}_{\mathrm{R}}(\mathrm{min})$ & Fatty Acid chain residues & Area \% \\
\hline 1 & 29.399 & Dodecanoic acid (C12:0) & 7.69 \\
2 & 34.316 & Tetradecanoic acid (C14:0) & 10.26 \\
4 & 38.299 & 9-Hexadecenoic acid (C16:1) & 5.86 \\
5 & 38.778 & Hexadecanoic acid (C16:0) & 24.94 \\
6 & 42.159 & $9,12-$ Octadecadienoic acid (Z,Z) (C18:2) & 6.62 \\
7 & 42.337 & 9-Octadecenoic acid (C18:1) & 36.46 \\
8 & 42.773 & Octadecanoic acid (C18:0) & 4.04 \\
\hline
\end{tabular}

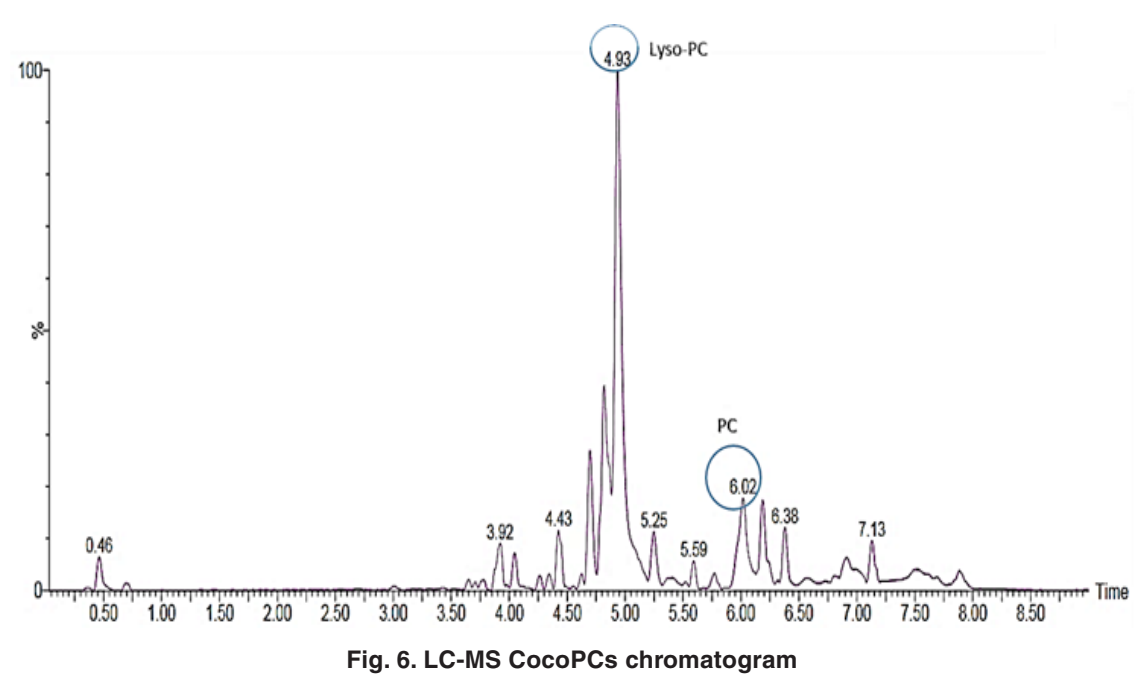

\section{CONCLUSION}

Approximately $6.317 \%$ of $100 \mathrm{mg}$ CocoPLs is CocoPCs. The isolated CocoPCs includes species with $\mathrm{m} / \mathrm{z} 763$ (16:0/18:0-PC) and $\mathrm{m} / \mathrm{z}$ 522 (18:1-LysoPC). The fatty acid chain residues of CocoPCs, with an abundance of more than
$4 \%$, are Dodecanoic acid (C12:0), Tetradecanoic acid (C14:0), 9-Hexadecenoic acid (C16:1), Hexadecanoic acid (C16:0), 9,12-Octadecadienoic acid (Z,Z) (C18:2), 9-Octadecenoic acid (C18:1) and Octadecanoic acid (C18:0), which corresponds to their presence in the chromatogram from low $t_{R}$ to high $t_{R}$. Further refinement is needed to obtain single component of CocoPCs. 


\section{ACKNOWLEDGEMENT}

$\mathrm{DH}$ and $\mathrm{KA}$ would like to express their gratitude for the financial support from DIPA FSM UNDIP 2017.

\section{REFERENCES}

1. Shrestha, H.; Bala, R.; Arora, S. J Pharm., 2014, 2014, 1-10.

2. Sakdiset, P.; Okada, A.; Todo, H.; Sugibayashi, K. J Drug Deliv Sci Technol., 2018, 44, (in press).

3. Hudiyanti, D.; Supardi, A.; Nugroho, S. J Sains Dan Mat., 2012, 20, 93-7.

4. Hudiyanti, D. Fosfolipida: Biosurfaktan. Yogyakarta: Deepublish., 2018, 17.

5. Deborah, F.; Sofia, M. M.; Romina, B.; Claudia, M.; Guido, F.; Gregorio, C.; Antonella, S.; Francesco, C.; Antonio, S. Phytomedicine ., 2018.

6. Tero R.; Fukumoto K.;MotegiT.; Yoshida M.; Niwano M.; Hirano-Iwata A. Sci Rep., 2017, 7, 17905.

7. Li J.; Wang X.; Zhang T.; Wang C.; Huang Z.; Luo X.; Deng Y. Asian J Pharm Sci., 2015, 10, 81-98.
8. Liu, D.; Ma, F. Soybean Phospholipids. In: Krezhova D, editor. Recent trends enhancing Divers. Qual. soybean Prod., InTech., 2011, 483-500.

9. Blesso, C. Nutrients., 2015, 7, 2731-47.

10. Hudiyanti, D.; Al Khafiz, M.F.; Anam, K. Orient J Chem., 2018, 34, 1136-40.

11. Hudiyanti, D.; Triana, D.; Siahaan, P. J Kim Sains Dan Apl., 2017, 20, 5-8.

12. Wojdyr, M. J Appl Crystallogr., 2010, 43, 1126-1128.

13. Swann G.E.A.; Patwardhan S. V. Clim Past., 2011, 7, 65-74.

14. Meng, X.; Pan, Q.; Ding, Y.; Jiang, L. Food Chem., 2014, 147, 272-278. 